\title{
Comparative performance of inflammation-based prognostic scores in patients operated for intrahepatic cholangiocarcinoma
}

This article was published in the following Dove Press journal: Cancer Management and Research

\author{
Jiajia $\operatorname{Lin}^{1, *}$ \\ Tingting Fang ${ }^{1} * *$ \\ Mengxuan Zhu',* \\ Xin Xu' \\ Juan Zhang' \\ Susu Zheng' \\ Chuyu Jing \\ Meixia Zhang' \\ Binbin Liu' \\ Boheng Zhang ${ }^{1,2}$ \\ 'The Liver Cancer Institute, Zhongshan \\ Hospital, Fudan University, Shanghai \\ 200032, People's Republic of China; \\ ${ }^{2}$ Center for Evidence-Based Medicine, \\ Fudan University, Shanghai 200032, \\ People's Republic of China
}

*These authors contributed equally to this work
Correspondence: Boheng Zhang; Binbin Liu The Liver Cancer Institute, Zhongshan Hospital, Fudan University, I 80 Fenglin Road, Xuhui District, Shanghai 200032,

People's Republic of China

Tel +86 2164038038

Fax +862164038038

Email zhang.boheng@zs-hospital.sh.cn;

liu.binbin@zs-hospital.sh.cn
Objective: Prognostic performance of inflammation-based prognostic scores, including the Glasgow Prognostic Score (GPS), modified Glasgow Prognostic Score (mGPS), neutrophil to lymphocyte ratio (NLR), platelet to lymphocyte ratio (PLR), lymphocyte to monocyte ratio (LMR), Prognostic Index (PI) and Prognostic Nutritional Index (PNI) has been explored in patients with varied types of cancer, though little data is available in intrahepatic cholangiocarcinoma (ICC). This study sought to evaluate the impact of systemic inflammation on the overall survival (OS) of ICC patients, and to identify more optimal prognostic indices.

Patients and methods: The prognostic power of all the scores mentioned above was compared in 123 patients underwent curative surgery for ICC using Kaplan-Meier curves, COX regression models and the receiver operating characteristics (ROC) curves. The results were validated in a cohort of 95 ICC patients.

Results: Multivariate analysis identified LMR as the only independent inflammation-based predictor for OS in the training cohort $(P=0.007$, HR 2.082, 95\% CI 1.218-3.558). More importantly, the combined score of LMR and pTNM designated the inflammation-based pathological stage (IPS) outperformed other established scores in terms of discriminatory ability, monotonicity and homogeneity in the training and validation cohorts.

Conclusion: This study reveals that preoperative LMR is an independent predictor of OS in ICC patients after hepatectomy, and the IPS can be applied as a novel prognostic indicator in these patients.

Keywords: inflammation-based prognostic score, the lymphocyte to monocyte ratio, intrahepatic cholangiocarcinoma, prognostic marker, staging system

\section{Introduction}

In the last three decades, having witnessed a marked increase in incidence ${ }^{1}$ as well as mortality rates ${ }^{2}$ globally, intrahepatic cholangiocarcinoma (ICC) remains the second most frequent primary liver cancer after hepatocellular carcinoma (HCC). Surgical resection is considered the only choice of potentially curative treatment for patients with operable ICC. Unfortunately, the surgical outcome is grim even after curative-intent resection, as the median survival period after hepatic resection is only 12.2 months. ${ }^{3}$ However, reports on the predictors of survival are relatively scarce.

Inflammation associated factors, such as hepatobiliary flukes, primary sclerosing cholangitis (PSC), chronic hepatitis $\mathrm{B} / \mathrm{C}$ infection and hepatolithiasis are known to be risk factors for ICC. They cause chronic biliary inflammation and increased 
cellular turnover. ${ }^{4}$ Moreover, the presence of systemic inflammation negatively correlates with prognosis of cancer patients. ${ }^{5,6}$

Previous studies have elucidated that preoperative inflammation-based prognostic scores are predictive of survival in patients with malignancies ${ }^{7-10}$ including ICC. ${ }^{11-13}$ These prognostic indices include the Glasgow Prognostic Score (GPS) and modified Glasgow Prognostic Score (mGPS) based on the serum concentrations of CRP and albumin, the Prognostic Index (PI) based on CRP concentration and white blood cell count, the neutrophil to lymphocyte ratio (NLR), the platelet to lymphocyte ratio (PLR), the lymphocyte to monocyte ratio (LMR), and the Prognostic Nutritional Index (PNI) based on albumin concentration and lymphocyte count. To our knowledge, no study has assessed the prognostic value of all these scores for ICC patients. In the present study, we compared the prognostic ability of these scores as well as pathological staging systems among patients who underwent radical resection for ICC to identify more optimal prognostic predictors for these patients.

\section{Materials and methods}

\section{Patients}

Medical records of ICC patients who underwent potentially curative hepatectomy at the Liver Cancer Institute of Zhongshan Hospital (Fudan University, Shanghai, China) between 2010 and 2014 were retrospectively reviewed. Curative hepatectomy was defined as the complete removal of all macroscopic tumor nodules with clear microscopic margins and no residual tumors as indicated by CT scan at one month after surgery. Only patients pathologically confirmed to have primary intrahepatic cholangiocarcinoma were enrolled in this study. Patients who underwent preoperative therapies like transarterial chemoembolization, radiofrequency ablation, or percutaneous ethanol injection and those who showed clinical evidence of infection or other inflammatory conditions were excluded. Patients with a history of other malignancies were also excluded. Finally a total of 218 patients with ICC were included and then randomly divided into two groups, termed the training cohort and validation cohort (123 and 95 patients, respectively). The pathological stage was classified according to the AJCC 7th edition. ${ }^{14}$ Blood samples were obtained 1-3 days before surgery for measurement of white blood cell count, neutrophil, lymphocyte, platelet (Plt), monocyte count, CRP, albumin, alkaline phosphatase (ALP), total bilirubin and other laboratory data relevant to this study.
This study complied with the standards of the Declaration of Helsinki and the current ethical guidelines, and was approved by the Zhongshan Hospital Ethics Committee. All data were analyzed and displayed anonymously, thus the identity of the study participants is concealed. Written informed consent for the use of clinicopathtological data for study purpose was obtained from participants on admission.

\section{Follow-up and postoperative treatment}

According to our routine follow-up procedure, patients were followed up monthly during the first postoperative year and every 3 months thereafter. Tumor markers, such as the CA19-9 and liver ultrasonography were assessed at each visit. A computed tomography (CT) scan of the abdomen was performed every 6 months. Bone scan or magnetic resonance imaging (MRI) was used when needed. If recurrence was suspected, additional investigations, such as hepatic angiography and positron emission tomography-computed tomography (PETCT) were performed. Patients with confirmed ICC recurrence received further treatment, if the recurrent tumor was localized, a second liver resection, radiofrequency ablation (RFA), or percutaneous ethanol injection (PEI) was suggested. If the recurrent tumor was multiple or diffused, patients were suggested to take transcatheter arterial chemoembolization (TACE) or chemotherapy; as for lymph node or bone metastasis, external radiotherapy was recommended. Overall survival (OS) was defined as the interval between the date of resection and the date of death or the last follow-up.

\section{Statistical analysis}

Continuous variables are presented as medians and ranges. Categorical variables are presented as numbers and percentages. Comparisons between groups were performed using the Student's unpaired $t$-test or Mann-Whitney $U$ test for continuous or ordinal variables, while Chi square test or Fisher's exact test was adopted for categorical variables, as appropriate. The overall survival rates were calculated using the Kaplan-Meier method, and differences in the survival rates between two groups were compared by the log-rank test. Backward stepwise multivariate Cox proportion analysis was performed to determine the influence of factors on OS which were significant in the univariate analysis.

A binary logistic regression model was fitted, and the regression coefficients derived from the model were used to create a new staging system as a composite score for LMR and pTNM, termed the inflammation-based pathological stage (IPS). To evaluate the discriminatory ability of each 
score, receiver operating characteristics (ROC) curves were generated, and the areas under the curve (AUC) were measured. The optimal cutoff was determined by ROC analysis. The monotonicity of each score was evaluated with the linear trend chi-square test, whereas the homogeneity of prognostic prediction across categories was measured using the likelihood ratio test as described in a previous study. ${ }^{15}$ The construction of the inflammation-based prognostic scores and their optimal cutoff values are shown in Table 1. Statistical analyses were performed using the SPSS statistical software package, version 17.0 (IBM SPSS Inc.,Chicago, IL, USA), at a significance level of $P$ less than 0.05 .

\section{Results}

\section{Clinicopathologic profile of patients}

The detailed baseline characteristics of patients in the training and validation cohorts are summarized in Table 2.

Table I Definition of inflammation-based prognostic scores

\begin{tabular}{|c|c|}
\hline Scoring systems & Score \\
\hline Glasgow Prognostic Score (GPS) & \\
\hline C-reactive protein $\leq 10 \mathrm{mg} / \mathrm{l}$ and albumin $\geq 35 \mathrm{~g} / \mathrm{l}$ & 0 \\
\hline C-reactive protein $\leq 10 \mathrm{mg} / \mathrm{l}$ and albumin $<35 \mathrm{~g} / \mathrm{l}$ & I \\
\hline C-reactive protein $>10 \mathrm{mg} / \mathrm{l}$ and albumin $\geq 35 \mathrm{~g} / \mathrm{l}$ & I \\
\hline C-reactive protein $>10 \mathrm{mg} / \mathrm{l}$ and albumin $<35 \mathrm{~g} / \mathrm{l}$ & 2 \\
\hline Modified Glasgow Prognostic Score (mGPS) & \\
\hline C-reactive protein $\leq 10 \mathrm{mg} / \mathrm{l}$ and albumin $\geq 35 \mathrm{~g} / \mathrm{l}$ & 0 \\
\hline C-reactive protein $\leq 10 \mathrm{mg} / \mathrm{l}$ and albumin $<35 \mathrm{~g} / \mathrm{l}$ & 0 \\
\hline C-reactive protein $>10 \mathrm{mg} / \mathrm{l}$ & I \\
\hline C-reactive protein $>10 \mathrm{mg} / \mathrm{l}$ and albumin $<35 \mathrm{~g} / \mathrm{l}$ & 2 \\
\hline Prognostic Index (PI) & \\
\hline C-reactive protein $\leq 10 \mathrm{mg} / \mathrm{l}$ and white cell count $\leq 11 \times 10^{9} / \mathrm{l}$ & 0 \\
\hline C-reactive protein $\leq 10 \mathrm{mg} / \mathrm{l}$ and white cell count $>11 \times 10^{9} / \mathrm{l}$ & 1 \\
\hline C-reactive protein $>10 \mathrm{mg} / \mathrm{l}$ and white cell count $\leq 11 \times 10^{9} / \mathrm{l}$ & I \\
\hline C-reactive protein $>10 \mathrm{mg} / \mathrm{l}$ and white cell count $>11 \times 10^{9} / \mathrm{l}$ & 2 \\
\hline Neutrophil to lymphocyte ratio (NLR) & \\
\hline Neutrophil count: lymphocyte count $<2.94$ & 0 \\
\hline Neutrophil count: lymphocyte count $\geq 2.94$ & I \\
\hline Platelet to lymphocyte ratio (PLR) & \\
\hline Platelet count: lymphocyte count $<130.59$ & 0 \\
\hline Platelet count: lymphocyte count $\geq 130.59$ & 1 \\
\hline Lymphocyte to monocyte ratio (LMR) & \\
\hline Lymphocyte count: monocyte count $<3.62$ & I \\
\hline Lymphocyte count: monocyte count $\geq 3.62$ & 0 \\
\hline Prognostic Nutritional Index (PNI) & \\
\hline Albumin $(g / L)+5 \times$ total lymphocyte count $\times 10^{9} / /<48.25$ & I \\
\hline Albumin $(g / L)+5 \times$ total lymphocyte count $\times 10^{9} / / \geq 48.25$ & 0 \\
\hline
\end{tabular}

Overall, there were no significant differences between the two cohorts with respect to all clinicopathological and demographic data as well as inflammation-based prognostic scores. Among the 218 patients enrolled in the study, 124 $(56.9 \%)$ were male. A total of $57(46.3 \%)$ patients in the training set and $45(47.4 \%)$ patients in the validation set died at the end of observation (March 2016). The median OS of the training cohort was 11.6 months (range 2.0-62.2 months) while that of the validation cohort was 16.8 months (range 1.3-62.7 months). No significant difference in OS between the two groups was noted.

\section{Univariate and multivariate analyses of prognostic factors for OS}

In the training set, univariate analysis revealed that ALP $(P=0.039)$, CA19-9 $(P=0.031)$, CEA $(P=0.013)$, multiple nodules $(P<0.001)$, maximal tumor diameter $(P=0.040)$, vascular invasion $(P=0.016)$, lymph node invasion $(P=0.006)$, pTNM stage $(P=0.001)$, the GPS $(P=0.027)$, mGPS $(P=0.025)$, PI $(P=0.020)$, NLR $(P=0.004)$, PLR $(P=0.009)$, $\operatorname{LMR}(P=0.005)$ and the PNI $(P=0.023)$ were risk factors for inferior overall survival. Multivariate analysis identified pTNM $(P=0.001$, HR $1.557,95 \%$ CI $1.200-2.022)$ and the LMR $(P=0.007$, HR 2.082, 95\% CI 1.218-3.558) as independent risk factors for poorer OS as shown in Table 3.

\section{Comparative prognostic performance of scoring systems}

The relationship between the prognostic scores and overall survival in the training set is shown in Figure 1. Higher levels of GPS, mGPS, NLR, PLR, LMR, PNI and pTNM were associated with reduced OS, although PI displayed marginal significance for predicting survival $(P=0.055)$. A combination score of LMR and pTNM, designated inflammationbased pathological stages (IPS) was constructed as shown in Table 4. Patients were grouped into three categories according to their IPS stages, which resulted in significant differences in OS between all adjacent strata. Compared with patients staged IPS I, those with IPS II in the training set were 2.641 times more likely to end up with inferior OS. For patients with IPS III, the relative hazard ratio was 4.955 (Table 4). In the validation set, only the PI, LMR, pTNM and the IPS were significantly related to overall survival rates (see Figure S1). Stepwise increase in IPS was accompanied with rise in hazard ratio in validation cohort.

To assess the discriminatory capacity of each scoring system, receiver operating characteristic curves were 
Table 2 Clinicopathtological characteristics of the patients in two cohorts

\begin{tabular}{|c|c|c|c|}
\hline Variable & $\begin{array}{l}\text { Training cohort } \\
(n=123)\end{array}$ & Validation cohort $(n=95)$ & $P$-value \\
\hline Age (years) & $60(31-85)$ & $61(37-79)$ & 0.476 \\
\hline Gender (Male/Female) & $65 / 58$ & $59 / 36$ & 0.171 \\
\hline $\operatorname{ALP}(I \cup / L)$ & $95(24-946)$ & $95(34-1280)$ & 0.507 \\
\hline Total serum bilirubin (mg/dL) & II (3.6-355.7) & II.4 (3.3-286.3) & 0.996 \\
\hline CAI9-9 (U/ml) & $49.4(0.6-10,000)$ & $46.8(0.6-10,000)$ & 0.807 \\
\hline CEA (ng/ml) & $2.8(0.5-945.2)$ & $3.3(0.5-133.3)$ & 0.440 \\
\hline $\mathrm{HBsAg}$ or HCV (positive/negative) & $46 / 77$ & $32 / 63$ & 0.571 \\
\hline Tumor number (solitary/multiple) & $80 / 43$ & $69 / 26$ & 0.232 \\
\hline Maximal tumour diameter $(\mathrm{cm})$ & $5.3(I-13.5)$ & $6(I-14)$ & 0.695 \\
\hline Liver cirrhosis (\%) & $18(14.6)$ & $21(22.1)$ & 0.154 \\
\hline Vascular invasion (absent/present) & $109 / 14$ & $83 / 12$ & 0.778 \\
\hline Microscopic vascular invasion (absent/present) & $95 / 28$ & $80 / 15$ & 0.199 \\
\hline Lymph node invasion (absent/present) & $109 / 14$ & $75 / 20$ & 0.051 \\
\hline Local extrahepatic invasion (absent/present)* & $111 / 12$ & $84 / 11$ & 0.664 \\
\hline PTNM (I/II/III/IV) & $49 / 50 / 10 / 14$ & $37 / 29 / 8 / 21$ & 0.272 \\
\hline Differentiation (well/moderate/poor) & $2 / 102 / 19$ & $\mathrm{I} / 77 / 17$ & 0.582 \\
\hline GPS $(0 / 1 / 2)$ & $94 / 26 / 3$ & $70 / 23 / 2$ & 0.662 \\
\hline Modified GPS (0/1/2) & $98 / 22 / 3$ & $75 / 18 / 2$ & 0.908 \\
\hline $\mathrm{PI}(0 / \mathrm{I} / 2)$ & $87 / 33 / 3$ & $70 / 24 / 1$ & 0.594 \\
\hline NLR $(0 / 1)$ & $69 / 54$ & $59 / 36$ & 0.372 \\
\hline $\operatorname{PLR}(0 / 1)$ & $60 / 63$ & $56 / 39$ & 0.136 \\
\hline $\operatorname{LMR}(0 / I)$ & $64 / 59$ & $49 / 46$ & 0.947 \\
\hline PNI $(0 / I)$ & $63 / 60$ & $56 / 39$ & 0.256 \\
\hline
\end{tabular}

Note: *Local extrahepatic invasion, tumor perforated the visceral peritoneum or involved the local extrahepatic structures by direct invasion.

Abbreviations: ALP, alkaline phosphatase; CAI9-9, carbohydrate antigen 19-9; CEA, carcinoembryonic antigen; HBsAg, hepatitis B surface antigen; HCV, hepatitis C virus; PTNM, pathological tumor-node-metastasis; GPS, Glasgow Prognostic Score; mGPS, modified Glasgow Prognostic Score; PI, Prognostic Index; NLR, neutrophil to lymphocyte ratio; PLR, platelet to lymphocyte ratio; LMR, lymphocyte to monocyte ratio; PNI, Prognostic Nutritional Index.

constructed for survival status at 12-month, 18-month and 24-month follow-up. The area under the ROC curve (AUC) was calculated and compared, as shown in Table 5. The IPS had the highest AUC value at 12-months (0.699), 18months (0.676) and 24-months (0.669) follow-up in comparison with other inflammation-based prognostic indices and the pTNM in the training set. This conclusion was furthere verified in the validation cohort. The IPS had an AUC value of $0.737,0.713,0.717$ at 12-month, 18-month and 24-month follow-up, respectively, as shown in Table S1. The IPS with the highest $\chi^{2}$ according to the linear trend test and the LR test was considered to have the best monotonicity and homogeneity, although pTNM stage appeared to have higher homogeneity in the training group as shown in Table 6 and Table S2.

\section{Relationship between clinicopathological factors and the IPS}

Several clinicopathological features were compared among the three groups with different level of IPS in the training cohort (Table 7). Those with the same elements that constituted pTNM or LMR including tumor number, maximal tumor diameter, lymph node invasion, vascular invasion, local extrahepatic invasion, PI, NLR, PLR and PNI were not compared. It turned out that patients with higher ALP, CEA, microscopic vascular invasion, elevated GPS and modified GPS were more likely to have advanced stages of IPS.

Similar conclusions were drawn in the validation cohort; in which the aforementioned factors were significantly associated with advanced IPS level in this set (Table S3).

\section{Discussion}

In the present study, we have demonstrated that inflammation-based prognostic scores such as GPS, mGPS, PI, NLR, PLR, LMR and PNI are associated with dismal prognosis in ICC patients underwent radical surgery. Besides, we report for the first time that LMR is an independent prognostic predictor of OS in these patients. 
Table 3 Univariate and multivariate analyses of prognostic factors

\begin{tabular}{|c|c|c|c|c|}
\hline \multirow[t]{2}{*}{ Variables } & \multirow[t]{2}{*}{$n=123$} & \multirow[t]{2}{*}{ Univariate $P$} & \multicolumn{2}{|c|}{ Multivariate analysis } \\
\hline & & & HR (95\% Cl) & $\mathbf{P}$ \\
\hline Age $(y r)(<65 / \geq 65)$ & $90 / 33$ & $0.84 I$ & & \\
\hline Gender (male/female) & $65 / 58$ & 0.593 & & \\
\hline ALP (IU/L, $<135 / \geq 135)$ & $96 / 27$ & 0.039 & & \\
\hline Total serum bilirubin $(\mathrm{mg} / \mathrm{dL}, \leq 20 />20)$ & $106 / 17$ & 0.860 & & \\
\hline CAI9-9 (U/ml, $\leq 37 />37)$ & $56 / 67$ & 0.031 & & \\
\hline CEA $(\mathrm{ng} / \mathrm{ml}, \leq 5 />5)$ & $90 / 33$ & 0.013 & & \\
\hline $\mathrm{HBsAg}$ or HCV (positive/negative) & $46 / 77$ & 0.204 & & \\
\hline Tumor number (solitary/multiple) & $80 / 43$ & $<0.001$ & & \\
\hline Maximal tumor diameter $(\mathrm{cm})(<5 / \geq 5)$ & $49 / 74$ & 0.040 & & \\
\hline Liver cirrhosis (absent/present) & $105 / 18$ & 0.509 & & \\
\hline Vascular invasion (absent/present) & $109 / 14$ & 0.016 & & \\
\hline Microscopic vascular invasion (absent/present) & $95 / 28$ & 0.099 & & \\
\hline Lymph node invasion (absent/present) & $109 / 14$ & 0.006 & & \\
\hline Local extrahepatic invasion (absent/present)* & $111 / 12$ & 0.387 & & \\
\hline pTNM (I/II+III+IV) & $49 / 74$ & 0.001 & $1.557(1.200-2.022)$ & 0.001 \\
\hline Differentiation (well/moderate/poor) & $2 / 102 / 19$ & 0.308 & & \\
\hline GPS $(0 / 1 / 2)$ & $94 / 26 / 3$ & 0.027 & & \\
\hline mGPS $(0 / 1 / 2)$ & $98 / 22 / 3$ & 0.025 & & \\
\hline $\mathrm{PI}(0 / 1 / 2)$ & $87 / 33 / 3$ & 0.020 & & \\
\hline $\operatorname{NLR}(0 / 1)$ & $69 / 54$ & 0.004 & & \\
\hline PLR $(0 / 1)$ & $60 / 63$ & 0.009 & & \\
\hline LMR $(0 / I)$ & $64 / 59$ & 0.005 & $2.082(1.218-3.558)$ & 0.007 \\
\hline PNI $(0 / I)$ & $63 / 60$ & 0.023 & & \\
\hline
\end{tabular}

Note: *Local extrahepatic invasion, tumor perforated the visceral peritoneum or involved the local extrahepatic structures by direct invasion.

Abbreviations: ALP, alkaline phosphatase; CAI9-9, carbohydrate antigen 19-9; CEA, carcinoembryonic antigen; HBsAg, hepatitis B surface antigen; HCV, hepatitis C virus; PTNM, pathological tumor-node-metastasis; GPS, Glasgow Prognostic Score; mGPS, modified Glasgow Prognostic Score; PI, Prognostic Index; NLR, neutrophil to lymphocyte ratio; PLR, platelet to lymphocyte ratio; LMR, lymphocyte to monocyte ratio; PNI, Prognostic Nutritional Index.

Based on this finding, we generated a novel inflammationbased prognostic score named the IPS, which proved to be more effective in stratifying the prognosis of patients with operable ICC.

Several lines of evidence indicate that inflammation acts much like "fertilizer" for the growth of malignancies. Inflammation is observed after activation of almost all common oncogenes including Myc and Ras ${ }^{16,17}$ and "is demonstrably capable of fostering the development of incipient neoplasias into full-blown cancers". ${ }^{18}$ Both local and systemic inflammatory responses are evident in the progression of human cancer. ${ }^{19,20}$ For intrahepatic cholangiocarcinoma, a previous study from our institution have validated that ICC cells are likely to recruit more neutrophils to the tumor foci through overexpressed CXCL5. ${ }^{21}$ Lin et al from Sun Yat-sen University Cancer Center demonstrated a significant association between high levels of serum CRP and adverse cancerspecific survival $(P=0.001)$ and recurrence-free survival $(P<0.001)^{22}$ in ICC. To assess the level of systemic inflammation in an effective way, several inflammation-based prognostic scores such as GPS, ${ }^{23} \mathrm{mGPS},{ }^{24} \mathrm{PI},{ }^{25} \mathrm{NLR},{ }^{26-28}$ $\mathrm{PLR}^{29-31} \mathrm{PNI}^{32-34}$ and LMR have been proposed in the recent decade. The prognostic power of these indices has been demonstrated in various types of malignancies including liver and bile duct neoplasm. ${ }^{7,9}$

However, few studies have looked into the prognostic ability of inflammation-based scores in patients with intrahepatic cholangiocarcinoma. Gomez et al reported, for the first time, that ICC patients with an elevated preoperative NLR $(>5)$ had significantly shorter overall survival after hepatic resection. ${ }^{11}$ Similarly, Chen et al found that high NLR $(\geq 2.49)$ showed notable correlation with early recurrence and poor overall survival in ICC patients. ${ }^{12}$ The same research group confirmed that high level of PLR $(\geq 123)$ might be a significant prognostic factor in ICC patients; PLR values greater than 123 reflected strong correlation with early recurrence and worse OS. ${ }^{13}$ In the present study, the prognostic significance of elevated PLR was only observed in the training set. However, PLR did not exert any significant effect on OS in the validation cohort, possibly due to limited cohort size. 
A
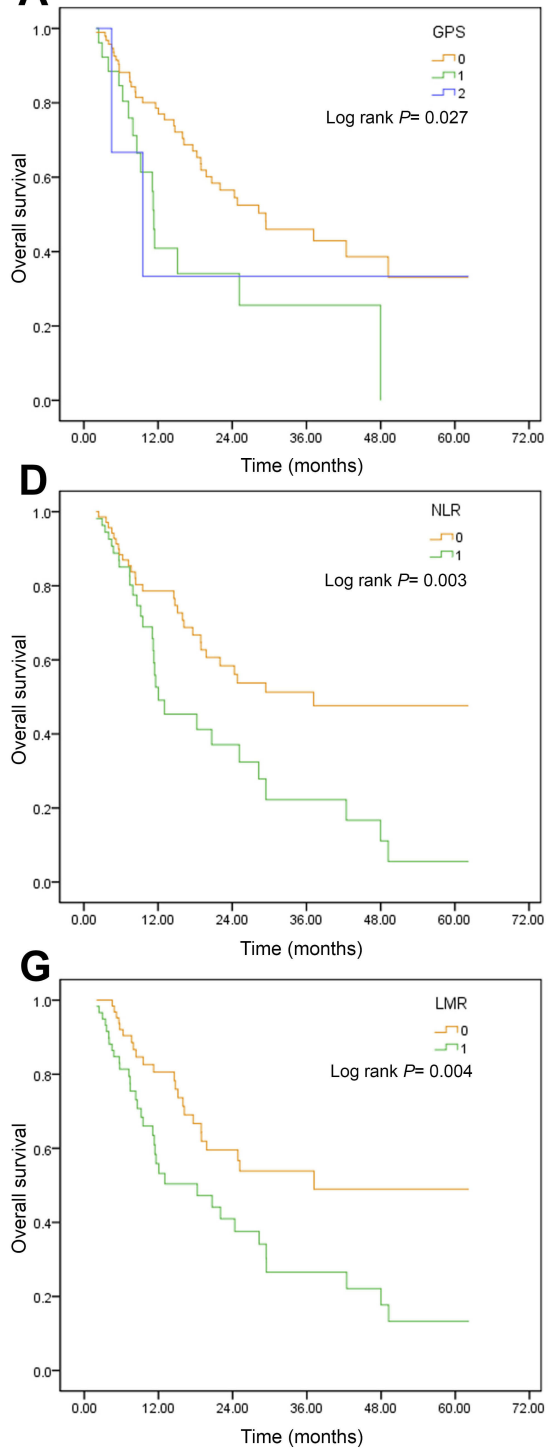

B
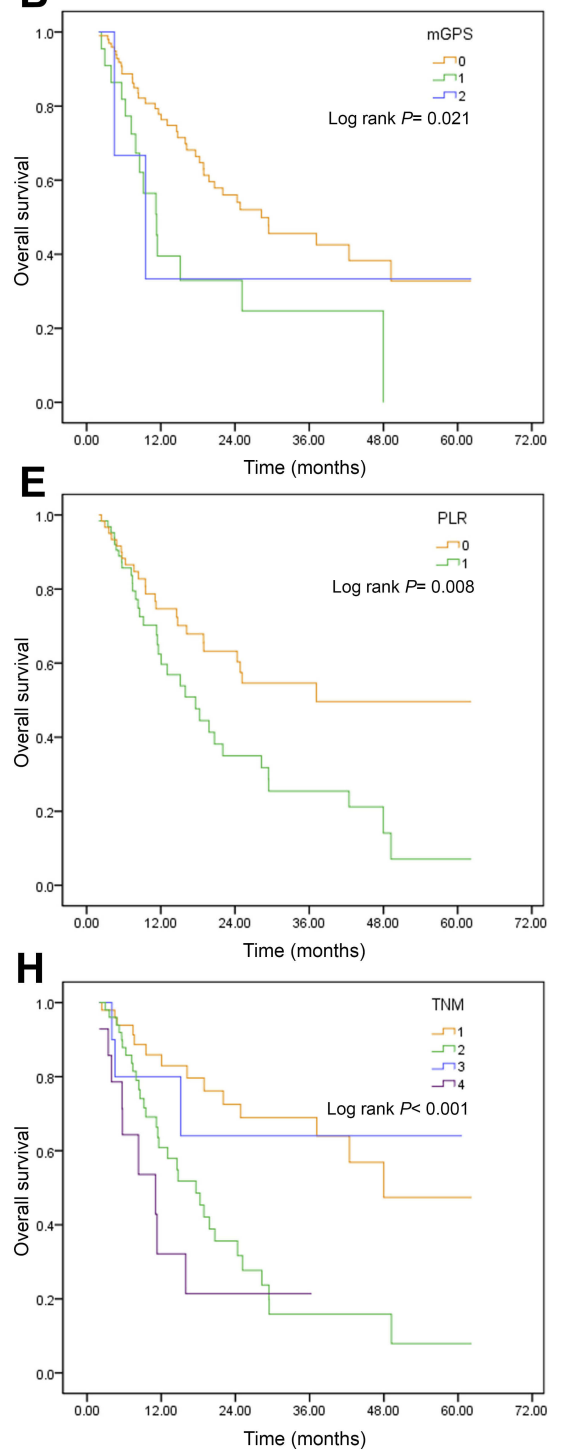

C

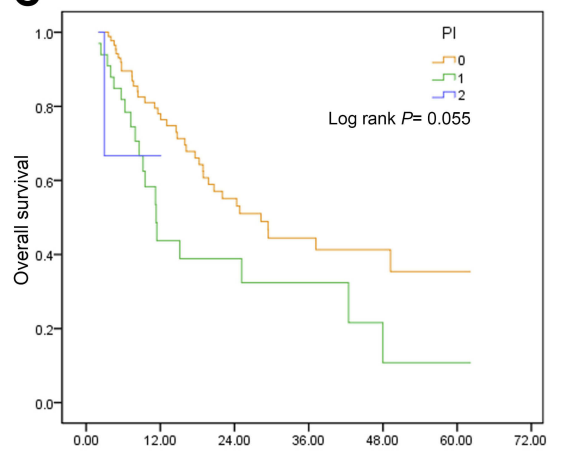

$\mathbf{F}$
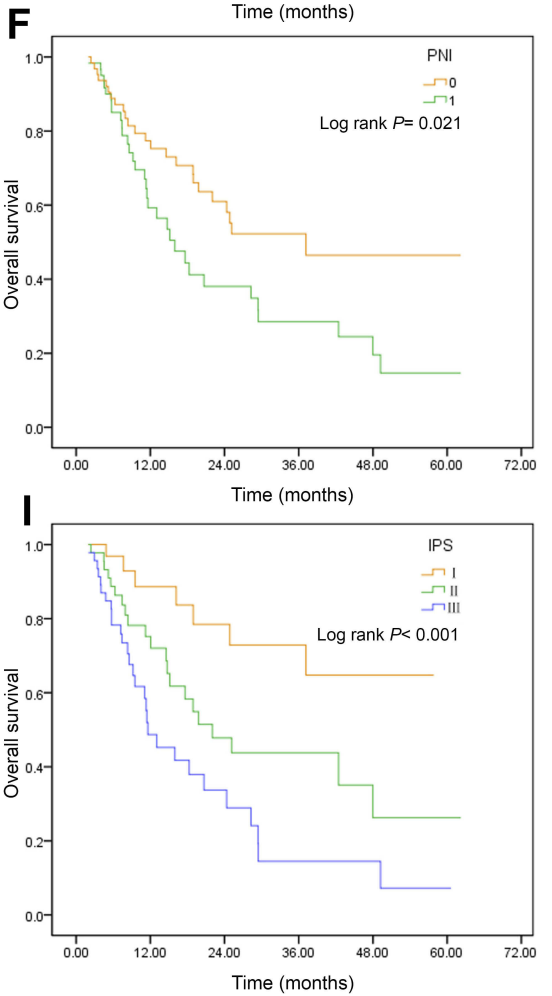

Figure I Kaplan-Meier overall survival curves for ICC patients undergoing curative hepatectomy stratified by inflammation-based prognostic scores and staging systems in the training cohort. (A) GPS; (B) mGPS; (C) PI; (D) NLR; (E) PLR; (F) PNI; (G) LMR; (H) PTNM; (I) IPS.

Abbreviations: GPS, Glasgow Prognostic Score; mGPS, modified Glasgow Prognostic Score; PI, Prognostic Index; NLR, neutrophil lymphocyte ratio; PLR, platelet (PIt) lymphocyte ratio; PNI, Prognostic Nutritional Index; LMR, lymphocyte monocyte ratio; PTNM, pathological tumor-node-metastasis; IPS, inflammation-based pathological stage.

Table 4 Construction of the inflammation-based pathological stage (IPS)

\begin{tabular}{|c|c|c|c|}
\hline \multirow[t]{2}{*}{ IPS = pTNM +2X LMR† } & \multirow[t]{2}{*}{ Stage } & \multicolumn{2}{|l|}{ Hazard ratio $(95 \% \mathrm{Cl}) \mathrm{P}$} \\
\hline & & Training set & Validation set \\
\hline IPS $=0$ or I & I & I & I \\
\hline IPS $=2$ or 3 & II & $2.64 I(I .12 I-6.222) 0.026$ & $2.591(1.070-6.275) 0.035$ \\
\hline IPS $=4$ or 5 or 6 & III & $4.955(2.154-11.399)<0.001$ & $5.539(2.375-12.916)<0.001$ \\
\hline
\end{tabular}

Note: $\nmid$ LMR was scored 0 or I according to Table I.

Abbreviations: PTNM, pathological tumor-node-metastasis; LMR, lymphocyte to monocyte ratio; $\mathrm{Cl}$, confidence interval.

In our comparative analysis of prognostic scores, the prognostic power of LMR, which had been tested by previous investigators, was also validated. Stotz et al demonstrated that elevated preoperative LMR was an independent predictor of increased TTR and OS in patients with stage II and III colon cancer. ${ }^{35}$ Lin and colleagues illustrated that the LMR was a significant prognostic factor for OS and DFS in patients receiving curative surgery for 
Table 5 Comparison of the AUC between inflammation-based prognostic scores in the training cohort

\begin{tabular}{|l|l|l|l|}
\hline & $\begin{array}{l}\text { Overall survival } \\
\text { AUc }\end{array}$ & Sensitivity & Specificity \\
\hline I2-Month & & & \\
GPS & 0.644 & 0.441 & \\
mGPS & 0.645 & 0.412 & 0.843 \\
PI & 0.621 & 0.471 & 0.775 \\
NLR & 0.583 & 0.559 & 0.607 \\
PLR & 0.553 & 0.588 & 0.517 \\
LMR & 0.636 & 0.676 & 0.596 \\
PNI & 0.569 & 0.588 & 0.551 \\
PTNM & 0.673 & 0.824 & 0.483 \\
IPS & 0.699 & 0.618 & 0.719 \\
\hline I8-Month & & & \\
GPS & 0.612 & 0.381 & 0.840 \\
mGPS & 0.618 & 0.357 & 0.877 \\
PI & 0.583 & 0.405 & 0.765 \\
NLR & 0.546 & 0.500 & 0.593 \\
PLR & 0.563 & 0.595 & 0.531 \\
LMR & 0.588 & 0.595 & 0.580 \\
PNI & 0.582 & 0.595 & 0.568 \\
PTNM & 0.675 & 0.810 & 0.506 \\
IPS & 0.676 & 0.548 & 0.716 \\
\hline 24-Month & & 0.333 & 0.827 \\
GPS & 0.581 & 0.312 & 0.867 \\
mGPS & 0.590 & 0.354 & 0.747 \\
PI & 0.548 & 0.604 & 0.587 \\
NLR & 0.533 & 0.583 \\
PLR & 0.575 & 0.587 \\
LMR & 0.585 & 0.561 & 0.562 \\
PNI & 0.659 & 0.520 \\
PTNM & IPS & & \\
\hline
\end{tabular}

Abbreviations: AUC, the area under the receiver operating characteristics curve; $\mathrm{Cl}$, confidence interval; GPS, Glasgow Prognostic Score; mGPS, modified Glasgow Prognostic Score; PI, Prognostic Index; NLR, neutrophil to lymphocyte ratio; PLR, platelet to lymphocyte ratio; LMR, lymphocyte to monocyte ratio; PNI, Prognostic Nutritional Index; pTNM, pathological tumor-node-metastasis; IPS, inflammationbased pathological stage.

hepatocellular carcinoma. ${ }^{36}$ Consistent with their conclusion, Wu et al stated that a high LMR may predict favorable OS and RFS in surgically treated HCC patients. ${ }^{37}$ In our study, we demonstrated for the first time that patients with increased pretreatment LMR ( $\geq 3.62)$ exhibited longer overall survival after hepatectomy for ICC. To the best of our knowledge, the prognostic performance of all these established inflammation-based prognostic scores has never been studied in a comparative fashion in intrahepatic cholangiocarcinoma. Our study not only confirmed the prognostic value of the GPS, mGPS, NLR, PLR, PNI,
Table 6 Evaluation of monotonicity and homogeneity of scoring systems in the training cohort

\begin{tabular}{|l|l|l|}
\hline Prognostic score & Linear trend test $\left(\chi^{2}\right)$ & LR test $\left(\chi^{2}\right)$ \\
\hline GPS & 3.594 & 3.815 \\
mGPS & 5.240 & 5.982 \\
PI & 1.047 & 2.388 \\
NLR & 2.081 & 2.101 \\
PLR & 2.997 & 3.035 \\
LMR & 5.761 & 5.851 \\
PNI & 3.503 & 3.548 \\
PTNM & 5.120 & 14.352 \\
IPS & 12.562 & 13.449 \\
\hline
\end{tabular}

Abbreviations: GPS, Glasgow Prognostic Score; mGPS, modified Glasgow Prognostic Score; PI, Prognostic Index; NLR, neutrophil to lymphocyte ratio; PLR, platelet to lymphocyte ratio; LMR, lymphocyte to monocyte ratio; PNI, Prognostic Nutritional Index; PTNM, pathological tumor-node-metastasis; IPS, inflammationbased pathological stage.

LMR and the PI but also highlighted the prominent prognostic value of LMR for operable ICC patients.

Components of these inflammation-based prognostic scores included in the present study comprise majority of the immune cells. Both innate and acquired immune cells play critical roles in the initiation, invasive growth and metastasis of cancer. Innate immune cells including macrophages, neutrophils, and mast cells are largely responsible for inflammatory reactions. ${ }^{38}$ In tumor sites, macrophages (and monocytes) create an inflammatory environment that is mutagenic and growth-promoting during tumor initiation. As tumors progress, they promote angiogenesis, enhance tumor cell migration, invasion and suppression of anti-tumor immunity. ${ }^{39}$ Macrophages at the tumor periphery can also foster local invasion by supplying matrix-degrading enzymes such as metalloproteinases and cysteine cathepsin proteases. ${ }^{18}$

The adaptive immune cells (B and T cells), however, can be tumor-suppressing. They carry out cancer immunosurveillance, recognize transformed cells and destroy them, resulting in a return to normal physiological tissue. ${ }^{40}$ A major subset of tumors shows evidence of a $\mathrm{T}$ cellinfiltrated phenotype. The presence of activated CD8+ T cells both within the tumor and in the peritumor microenvironment has been reported to indicate positive prognosis. ${ }^{41,42}$ Therefore, it is conceivable that higher LMR, as a compound outcome of increased peripheral blood lymphocytes and diminished monocytes, indicates favorable prognosis.

Clinicopathological predictors have proved to be suboptimal in identifying high-risk patients. But recent evidence underscored the discriminatory power of combined 
Table 7 Clinicopathtological characteristics of the patients grouped according to IPS in the training cohort

\begin{tabular}{|c|c|c|c|c|}
\hline Variable & $\begin{array}{l}\text { IPS I } \\
(n=32)\end{array}$ & $\begin{array}{l}\text { IPS II } \\
(n=45)\end{array}$ & $\begin{array}{l}\text { IPS III } \\
(n=46)\end{array}$ & $P$-value \\
\hline Age (yr), $(<65 / \geq 65)$ & $25 / 7$ & $34 / 11$ & $31 / 15$ & 0.268 \\
\hline Gender (Male/Female) & $17 / 15$ & $22 / 23$ & $26 / 20$ & 0.692 \\
\hline $\operatorname{ALP}(I U / L,<135 / \geq 135)$ & $28 / 4$ & $38 / 7$ & $30 / 16$ & 0.012 \\
\hline Total serum bilirubin $(\mathrm{mg} / \mathrm{dL}, \leq 20 />20)$ & $28 / 4$ & $40 / 5$ & $38 / 8$ & 0.477 \\
\hline CAI9-9 $(\mathrm{U} / \mathrm{ml}, \leq 37 />37)$ & $16 / 16$ & $22 / 23$ & $18 / 28$ & 0.307 \\
\hline CEA $(\mathrm{ng} / \mathrm{ml}, \leq 5 />5)$ & $26 / 6$ & $37 / 8$ & $27 / 19$ & 0.014 \\
\hline $\mathrm{HBsAg}$ or HCV (positive/negative) & $19 / 13$ & $11 / 34$ & $16 / 30$ & 0.070 \\
\hline Liver cirrhosis (absent/present) & $25 / 7$ & $42 / 3$ & $38 / 8$ & 0.810 \\
\hline Microscopic vascular invasion (absent/present) & $32 / 0$ & $37 / 8$ & $26 / 20$ & $<0.001$ \\
\hline Differentiation (well+moderate/poor) & $29 / 3$ & $36 / 9$ & $39 / 7$ & 0.602 \\
\hline GPS $(0 / 1+2)$ & $30 / 2$ & $31 / 14$ & $33 / 13$ & 0.048 \\
\hline Modified GPS $(0 / 1+2)$ & $31 / 1$ & $33 / 12$ & $34 / 12$ & 0.026 \\
\hline
\end{tabular}

Abbreviations: ALP, alkaline phosphatase; CAI9-9, carbohydrate antigen 19-9; CEA, carcinoembryonic antigen; HBsAg, hepatitis B surface antigen; HCV, hepatitis C virus; GPS, Glasgow rognostic score; mGPS, modified glasgow prognostic score; IPS, inflammation-based pathological stage.

prognostic index of the clinicopathological predictors like staging systems and the inflammation based indices. Pinato et al proposed a new prognostic score based on a combination of mGPS and CLIP scores. They found that the predictive accuracy of the combined score (c score 0.7 , 95\% CI 0.6-0.8) appeared to be superior to that of the CLIP score alone (c score $0.6,95 \%$ CI $0.5-0.7$ ). ${ }^{43}$ Kinoshita and co-workers also elucidated that when GPS was combined with the CLIP system to form a new prognostic system, named inflammation-based CLIP, better prognostic accuracy was achieved compared to GPS or CLIP alone. ${ }^{44}$ Unlike hepatocellular carcinoma, there are barely established staging systems for ICC. The most commonly used staging system for ICC is the TNM classification system. In this study, we verified the prognostic power of the inflammation-based pathological stage (IPS). It seems that the IPS is superior to the pTNM staging system and the inflammation-based indices alone in terms of discriminatory power (large differences in prognosis between different stages), homogeneity (small differences in prognosis between patients in the same stage) and monotonicity (mortality of patients increased significantly with the increase of staging). Kaplan-Meier curve revealed that patients with advanced IPS stages, even in the same pTNM stage of ICC when undergoing operation, got inferior overall survival rates. The stratification of prognosis according to the IPS stages was definitive, which totally outperformed the traditional pTNM staging system. In addition, IPS scores were strongly linked to tumor markers including ALP, CEA, and aggressive pathological characteristics like microscopic vascular invasion, all of which reflect poor prognosis. This indicates that more intense follow-up or prophylactic postoperative treatment such as chemotherapy, radiotherapy and transcatheter arterial chemoembolization (TACE) is needed for patients with advanced IPS stages.

In summary, this study reveals that the GPS, mGPS, NLR, PLR, PNI, LMR and the PI possess high prognostic value for OS in operable ICC. LMR was identified as the only independent predictor of OS among those inflammation-based scores. Besides, a novel and powerful inflammation-based prognostic index termed the IPS was established. Given the retrospective, small size and single institution-based nature of this study, certain limitations regarding this prognostic analysis should be acknowledged. Therefore, the findings of this study should be independently validated through prospective multicentric large cohort studies in the future.

\section{Acknowledgments}

This work is supported by the National Natural Science Foundation of China (Grant No. 81173391), Shanghai Municipal Commission of Health and Family Planning (ZY3-CCCX-3-2004) and Zhongshan Hospital Science Foundation (2018ZSQN35). The funding sources did not have any role in study design, data collection and analysis, decision to publish.

\section{Disclosure}

Dr Boheng Zhang reports grants from National Natural Science Foundation of China, Shanghai Municipal Commission of Health and Family Planning, during the 
conduct of the study. Dr Jiajia Lin reports grant from Zhongshan Hospital Science Foundation, during the conduct of the study. The authors declare no other conflicts of interest in this work.

\section{References}

1. Shaib YH, Davila JA, McGlynn K, El-Serag HB. Rising incidence of intrahepatic cholangiocarcinoma in the United States: a true increase? J Hepatol. 2004;40:472-477. doi:10.1016/j.jhep.2003.11.030

2. Patel T. Worldwide trends in mortality from biliary tract malignancies. BMC Cancer. 2002;2:10. doi:10.1186/1471-2407-2-10

3. Zou S, Li J, Zhou H, et al. Mutational landscape of intrahepatic cholangiocarcinoma. Nat Commun. 2014;5:5696. doi:10.1038/ ncomms6696

4. Bridgewater J, Galle PR, Khan SA, et al. Guidelines for the diagnosis and management of intrahepatic cholangiocarcinoma. $J$ Hepatol. 2014;60:1268-1289. doi:10.1016/j.jhep.2014.01.021

5. Hashimoto K, Ikeda Y, Korenaga D, et al. The impact of preoperative serum C-reactive protein on the prognosis of patients with hepatocellular carcinoma. Cancer-Am Cancer Soc. 2005;103:1856-1864.

6. McMillan DC, Canna K, McArdle CS. Systemic inflammatory response predicts survival following curative resection of colorectal cancer. Br J Surg. 2003;90:215-219. doi:10.1002/bjs.4038

7. Kinoshita A, Onoda H, Imai N, et al. Comparison of the prognostic value of inflammation-based prognostic scores in patients with hepatocellular carcinoma. Br J Cancer. 2012;107:988-993. doi:10.1038/bjc.2012.354

8. Pinato DJ, Shiner RJ, Seckl MJ, Stebbing J, Sharma R, Mauri FA. Prognostic performance of inflammation-based prognostic indices in primary operable non-small cell lung cancer. Brit $J$ Cancer. 2014;110:1930-1935. doi:10.1038/bjc.2014.145

9. Okuno M, Ebata T, Yokoyama Y, et al. Evaluation of inflammationbased prognostic scores in patients undergoing hepatobiliary resection for perihilar cholangiocarcinoma. J Gastroenterol. 2016;51:153161. doi:10.1007/s00535-015-1103-y

10. Wang D, Luo H, Qiu M, et al. Comparison of the prognostic values of various inflammation based factors in patients with pancreatic cancer. Med Oncol. 2012;29:3092-3100. doi:10.1007/s12032-012-0226-8

11. Gomez D, Morris-Stiff G, Toogood GJ, Lodge JPA, Prasad KR. Impact of systemic inflammation on outcome following resection for intrahepatic cholangiocarcinoma. J Surg Oncol. 2008;97:513518. doi:10.1002/jso.21001

12. Chen Q, Yang L, Li X, et al. The elevated preoperative neutrophil-tolymphocyte ratio predicts poor prognosis in intrahepatic cholangiocarcinoma patients undergoing hepatectomy. Tumor Biol. 2015;36:5283-5289. doi:10.1007/s13277-015-3188-6

13. Chen Q, Dai Z, Yin D, et al. Negative impact of preoperative platelet-lymphocyte ratio on outcome after hepatic resection for intrahepatic cholangiocarcinoma. Medicine. 2015;94:e574. doi:10.1097/MD.0000000000000874

14. Edge SB BD CCFA. AJCC Cancer Staging Manual. 7th ed. New York: Springer; 2010.

15. Ueno S. Discrimination value of the new western prognostic system (CLIP score) for hepatocellular carcinoma in 662 Japanese patients. HEPATOLOGY. 2001;34:529-534. doi:10.1053/jhep.2001.27219

16. Soucek L, Lawlor ER, Soto D, Shchors K, Swigart LB, Evan GI. Mast cells are required for angiogenesis and macroscopic expansion of Myc-induced pancreatic islet tumors. Nat Med. 2007;13:12111218. doi: $10.1038 / \mathrm{nm} 1649$

17. Sparmann A, Bar-Sagi D. Ras-induced interleukin-8 expression plays a critical role in tumor growth and angiogenesis. Cancer Cell. 2004;6:447-458. doi:10.1016/j.ccr.2004.09.028

18. Hanahan D, Weinberg RA. Hallmarks of cancer: the next generation. Cell. 2011;144:646-674. doi:10.1016/j.cell.2011.02.013
19. Roxburgh CS, Salmond JM, Horgan PG, Oien KA, McMillan DC. Comparison of the prognostic value of inflammation-based pathologic and biochemical criteria in patients undergoing potentially curative resection for colorectal cancer. Ann Surg. 2009;249:788-793. doi:10.1097/SLA.0b013e3181a3e738

20. Canna K, McArdle PA, McMillan DC, et al. The relationship between tumour T-lymphocyte infiltration, the systemic inflammatory response and survival in patients undergoing curative resection for colorectal cancer. Br J Cancer. 2005;92:651-654. doi:10.1038/sj.bjc.6602419

21. Zhou SL, Dai Z, Zhou ZJ, et al. CXCL5 contributes to tumor metastasis and recurrence of intrahepatic cholangiocarcinoma by recruiting infiltrative intratumoral neutrophils. Carcinogenesis. 2014;35:597-605. doi:10.1093/carcin/bgt397

22. Lin ZY, Liang ZX, Zhuang PL, et al. Intrahepatic cholangiocarcinoma prognostic determination using pre-operative serum $\mathrm{C}$-reactive protein levels. BMC Cancer. 2016;16:792. doi:10.1186/s12885-016-2827-7

23. Forrest LM, McMillan DC, McArdle CS, Angerson WJ, Dunlop DJ. Evaluation of cumulative prognostic scores based on the systemic inflammatory response in patients with inoperable non-small-cell lung cancer. $B r J$ Cancer. 2003;89:1028-1030. doi:10.1038/sj. bjc. 6601242

24. Proctor MJ, Morrison DS, Talwar D, et al. An inflammation-based prognostic score (mGPS) predicts cancer survival independent of tumour site: a Glasgow Inflammation Outcome Study. Br J Cancer. 2011;104:726-734. doi:10.1038/sj.bjc.6606087

25. Proctor MJ, Morrison DS, Talwar D, et al. A comparison of inflammation-based prognostic scores in patients with cancer. A Glasgow Inflammation Outcome Study. Eur J Cancer. 2011;47:2633-2641. doi:10.1016/j.ejca.2011.03.028

26. Mano Y, Shirabe K, Yamashita Y, et al. Preoperative neutrophil-tolymphocyte ratio is a predictor of survival after hepatectomy for hepatocellular carcinoma. Ann Surg. 2013;258:301-305. doi:10.1097/ SLA.0b013e318297ad6b

27. Jung J, Park SY, Park S, Park J. Prognostic value of the neutrophil-tolymphocyte ratio for overall and disease-free survival in patients with surgically treated esophageal squamous cell carcinoma. Tumor Biol. 2015;37:7149-7154. doi:10.1007/s13277-015-4596-3

28. Shimada H, Takiguchi N, Kainuma $\mathrm{O}$, et al. High preoperative neutrophil-lymphocyte ratio predicts poor survival in patients with gastric cancer. Gastric Cancer. 2010;13:170-176. doi:10.1007/ s10120-010-0554-3

29. Koh C, Bhoo-Pathy N, Ng K, et al. Utility of pre-treatment neutrophil-lymphocyte ratio and platelet-lymphocyte ratio as prognostic factors in breast cancer. Brit $J$ Cancer. 2015;113:150-158. doi:10.1038/bjc. 2015.183

30. Zhang Y, Jiang C, Li J, Sun J, Qu X. Prognostic significance of preoperative neutrophil/lymphocyte ratio and platelet/lymphocyte ratio in patients with gallbladder carcinoma. Clin Transl Oncol. 2015;17:810-818. doi:10.1007/s12094-015-1310-2

31. Jiang N, Deng J, Liu Y, Ke B, Liu H, Liang H. The role of preoperative neutrophil-lymphocyte and platelet-lymphocyte ratio in patients after radical resection for gastric cancer. Biomarkers. 2014;19:444-451. doi:10.3109/1354750X.2014.926567

32. Chan AWH, Chan SL, Wong GLH, et al. Prognostic Nutritional Index (PNI) predicts tumor recurrence of very early/early stage hepatocellular carcinoma after surgical resection. Ann Surg Oncol. 2015;22:4138-4148. doi:10.1245/s10434-015-4516-1

33. Pinato DJ, North BV, Sharma R. A novel, externally validated inflammation-based prognostic algorithm in hepatocellular carcinoma: the prognostic nutritional index (PNI). $\mathrm{Br} J$ Cancer. 2012;106:1439-1445. doi:10.1038/bjc.2012.92

34. Okamura Y, Ashida R, Ito T, Sugiura T, Mori K, Uesaka K. Preoperative neutrophil to lymphocyte ratio and prognostic nutritional index predict overall survival after hepatectomy for hepatocellular carcinoma. World J Surg. 2015;39:1501-1509. doi:10.1007/ s00268-015-2982-z 
35. Stotz M, Pichler M, Absenger G, et al. The preoperative lymphocyte to monocyte ratio predicts clinical outcome in patients with stage III colon cancer. Brit J Cancer. 2013;110:435-440. doi:10.1038/ bjc. 2013.785

36. Lin Z. Lymphocyte-to-monocyte ratio predicts survival of patients with hepatocellular carcinoma after curative resection. World $J$ Gastroentero. 2015;21:10898. doi:10.3748/wjg.v21.i38.10898

37. Wu S, Lin Y, Ye H, Li F, Xiong X, Cheng N. Lymphocyte to monocyte ratio and prognostic nutritional index predict survival outcomes of hepatitis B virus-associated hepatocellular carcinoma patients after curative hepatectomy. J Surg Oncol. 2016;114:202210. doi:10.1002/jso. 24297

38. Szabo G, Mandrekar P, Dolganiuc A. Innate immune response and hepatic inflammation. Semin Liver Dis. 2007;27:339-350. doi:10.1055/s-2007991511

39. Qian BZ, Pollard JW. Macrophage diversity enhances tumor progression and metastasis. Cell. 2010;141:39-51. doi:10.1016/j.cell.20 10.03 .014
40. Dunn GP, Old L, Schreiber RD. The immunobiology of cancer immunosurveillance and immunoediting. Annu Rev Immunol. 2004;21:137-148.

41. Hamanishi J, Mandai M, Iwasaki M, et al. Programmed cell death 1 ligand 1 and tumor-infiltrating CD8+ T lymphocytes are prognostic factors of human ovarian cancer. Proc Natl Acad Sci U S A. 2007;104:3360-3365. doi:10.1073/pnas.0611533104

42. Galon J. Type, density, and location of immune cells within human colorectal tumors predict clinical outcome. SCIENCE. 2006;313:19601964. doi:10.1126/science.1129139

43. Pinato DJ, Stebbing J, Ishizuka M, et al. A novel and validated prognostic index in hepatocellular carcinoma: the inflammation based index (IBI). J Hepatol. 2012;57:1013-1020. doi:10.1016/j. jhep.2012.06.022

44. Kinoshita A, Onoda H, Imai N, et al. The glasgow prognostic score, an inflammation based prognostic score, predicts survival in patients with hepatocellular carcinoma. BMC Cancer. 2013;13:52. doi:10.1186/14712407-13-52 


\section{Supplemantary materials}

Table SI Comparison of the AUC between between inflammation-based prognostic scores in the validation cohort

\begin{tabular}{|l|l|l|l|}
\hline & $\begin{array}{l}\text { Overall survival } \\
\text { AUC }\end{array}$ & Sensitivity & Specificity \\
\hline I2-Month & & & \\
GPS & 0.584 & 0.379 & 0.788 \\
mGPS & 0.597 & 0.345 & 0.848 \\
PI & 0.612 & 0.414 & 0.803 \\
NLR & 0.575 & 0.483 & 0.667 \\
PLR & 0.552 & 0.483 & 0.621 \\
LMR & 0.598 & 0.621 & 0.576 \\
PNI & 0.602 & 0.552 & 0.652 \\
PTNM & 0.728 & 0.862 & 0.500 \\
IPS & 0.737 & 0.690 & 0.682 \\
\hline I8-Month & & & \\
GPS & 0.608 & 0.395 & 0.825 \\
mGPS & 0.630 & 0.368 & 0.895 \\
PI & 0.655 & 0.447 & 0.860 \\
NLR & 0.623 & 0.526 & 0.719 \\
PLR & 0.575 & 0.500 & 0.649 \\
LMR & 0.645 & 0.658 & 0.632 \\
PNI & 0.596 & 0.526 & 0.667 \\
PTNM & 0.657 & 0.763 & 0.491 \\
IPS & 0.713 & 0.632 & 0.702 \\
\hline 24-Month & & 0.341 & 0.804 \\
GPS & 0.571 & 0.386 & 0.843 \\
mGPS & 0.598 & 0.455 & 0.686 \\
PI & 0.617 & 0.455 & 0.627 \\
NLR & 0.570 & 0.614 & 0.627 \\
PLR & 0.541 & 0.477 & 0.647 \\
LMR & 0.621 & 0.750 & 0.510 \\
PNI & 0.562 & 0.725 \\
PTNM & 0.677 & 0.717 & \\
IPS & & & \\
\hline
\end{tabular}

Abbreviations: AUC, the area under the receiver operating characteristics curve; $\mathrm{Cl}$, confidence interval; GPS, Glasgow Prognostic Score; mGPS, modified Glasgow Prognostic Score; PI, Prognostic Index; NLR, neutrophil to lymphocyte ratio; PLR, platelet (PIt)to lymphocyte ratio; LMR, lymphocyte to monocyte ratio; PNI, Prognostic Nutritional Index; PTNM, pathological tumor-node-metastasis; IPS, inflammation-based pathological stage.
Table S2 Evaluation of monotonicity and homogeneity of scoring systems in the validation cohort

\begin{tabular}{|l|l|l|}
\hline Prognostic score & Linear trend test $\left(\chi^{2}\right)$ & LR test $\left(\chi^{2}\right)$ \\
\hline GPS & 0.326 & 0.703 \\
mGPS & 0.994 & 1.959 \\
PI & 3.028 & 3.533 \\
NLR & 1.810 & 1.852 \\
PLR & 0.59 & 0.60 \\
LMR & 4.931 & 5.041 \\
PNI & 0.357 & 0.361 \\
PTNM & 6.684 & 8.668 \\
IPS & 11.588 & 12.008 \\
\hline
\end{tabular}

Abbreviations: GPS, Glasgow Prognostic Score; mGPS, modified Glasgow Prognostic Score; PI, Prognostic Index; NLR, neutrophil to lymphocyte ratio; PLR, platelet (PIt)to lymphocyte ratio; LMR, lymphocyte to monocyte ratio; PNI, Prognostic Nutritional Index; PTNM, pathological tumor-node-metastasis; IPS, inflammation-based pathological stage. 
Table S3 Clinicopathtological characteristics of the patients grouped according to the IPS in the validation cohort

\begin{tabular}{|c|c|c|c|c|}
\hline Variable & $\begin{array}{l}\text { IPS I } \\
(n=22)\end{array}$ & $\begin{array}{l}\text { IPS II } \\
(n=32)\end{array}$ & $\begin{array}{l}\text { IPS III } \\
(n=4 I)\end{array}$ & $P$-value \\
\hline Age $(y r),(<65 / \geq 65)$ & $12 / 10$ & $20 / 12$ & $24 / 17$ & 0.871 \\
\hline Gender (Male/Female) & $15 / 7$ & $19 / 13$ & $25 / 16$ & 0.663 \\
\hline $\operatorname{ALP}(I U / L,<|35 / \geq| 35)$ & $22 / 0$ & $25 / 7$ & $28 / 13$ & 0.005 \\
\hline Total serum bilirubin $(\mathrm{mg} / \mathrm{dL}, \leq 20 />20)$ & $21 / 1$ & $29 / 3$ & $33 / 8$ & 0.070 \\
\hline CAI99 (U/ml, $\leq 37 />37)$ & $15 / 7$ & $12 / 20$ & $19 / 22$ & 0.236 \\
\hline CEA (ng/ml, $\leq 5 />5)$ & $20 / 2$ & $24 / 8$ & $27 / 14$ & 0.035 \\
\hline $\mathrm{HBsAg}$ or HCV (positive/negative) & $9 / 13$ & $|1 / 2|$ & $12 / 29$ & 0.357 \\
\hline Liver cirrhosis(absent/present) & $17 / 5$ & $23 / 9$ & $34 / 7$ & 0.447 \\
\hline Microscopic vascular invasion (absent/present) & $22 / 0$ & $27 / 5$ & $31 / 10$ & 0.015 \\
\hline Differentiation (well+moderate/poor) & $19 / 3$ & $25 / 7$ & $34 / 7$ & 0.892 \\
\hline GPS $(0 / 1+2)$ & $20 / 2$ & $25 / 7$ & $25 / 16$ & 0.008 \\
\hline Modified GPS $(0 / 1+2)$ & $21 / 1$ & $26 / 6$ & $28 / 13$ & 0.012 \\
\hline
\end{tabular}

Abbreviations: ALP, alkaline phosphatase; GGT, Gamma Glutamyl Transferase; CAI9-9, carbohydrate antigen 19-9; CEA, carcinoembryonic antigen; HBsAg, hepatitis B surface antigen; HCV, hepatitis C virus; GPS, Glasgow Prognostic Score; mGPS, modified Glasgow Prognostic Score; IPS, inflammation-based pathological stage. 
A
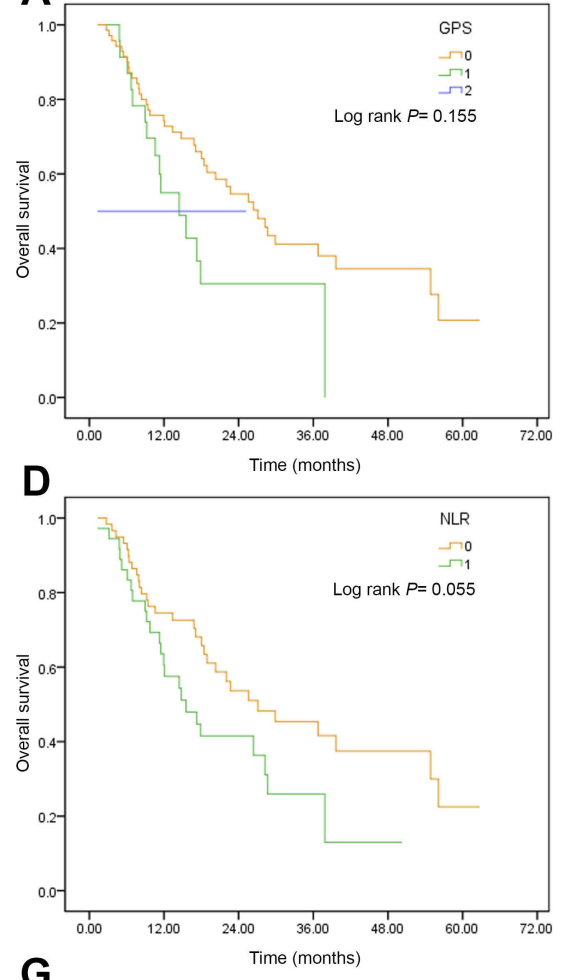

G

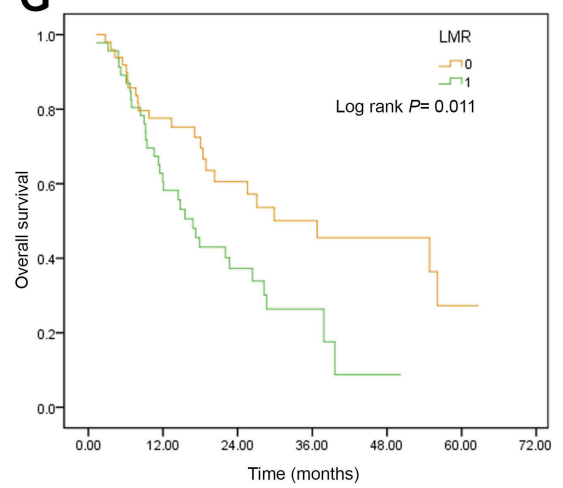

B

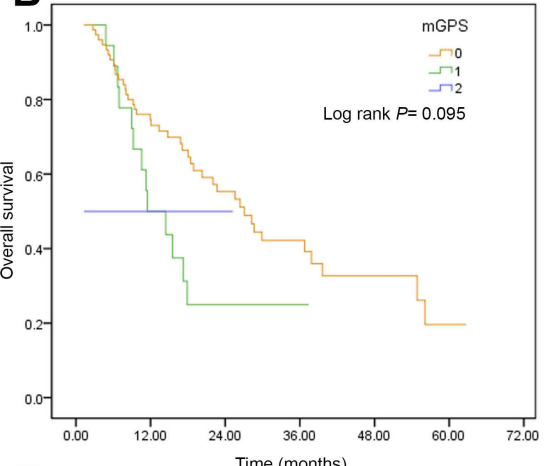

E

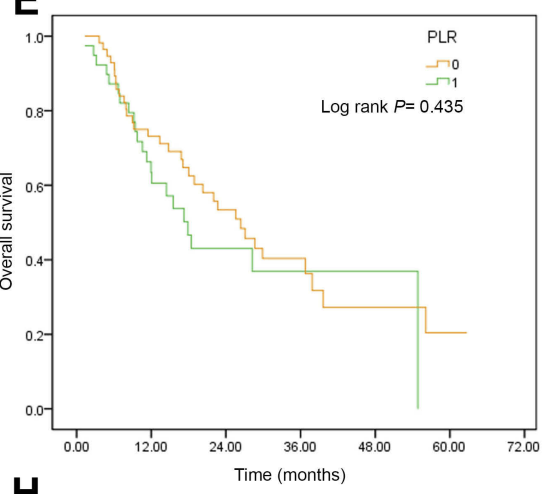

$\mathbf{H}$

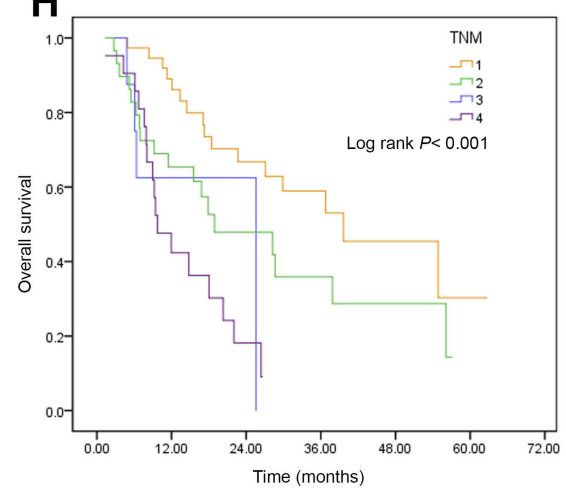

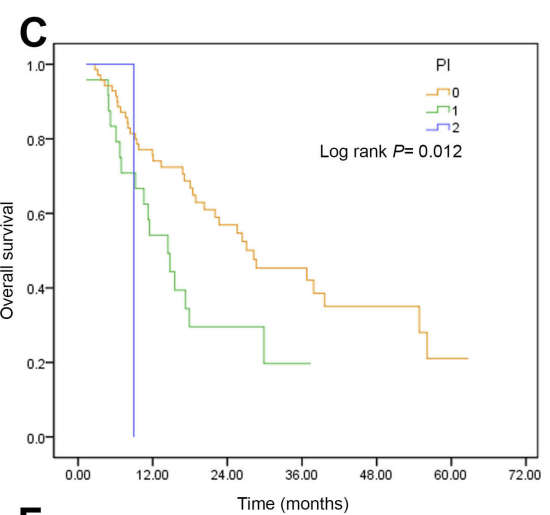

$\mathbf{F}$

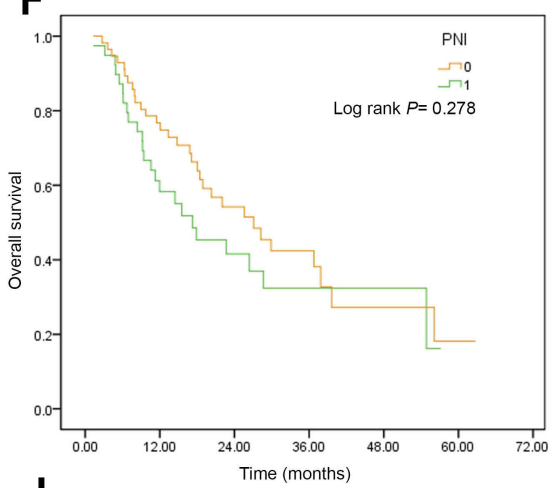

I

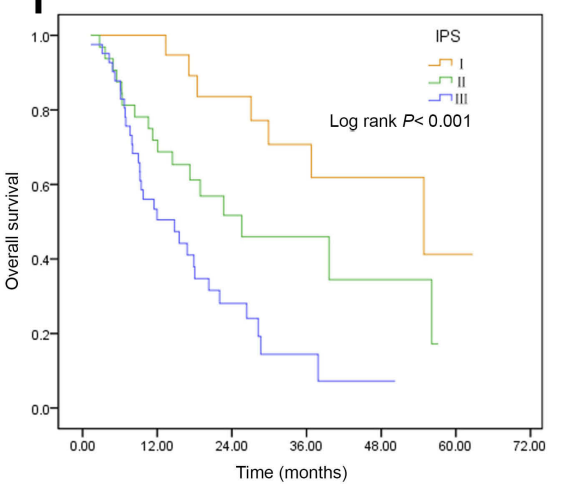

Figure SI Kaplan-Meier overall survival curves for ICC patients undergoing curative hepatectomy stratified by inflammation-based prognostic scores and staging systems in the validation cohort. (A) GPS; (B) mGPS; (C) PI; (D) NLR; (E) PLR; (F) PNI; (G) LMR; (H) PTNM; (I) IPS.

\section{Publish your work in this journal}

Cancer Management and Research is an international, peer-reviewed open access journal focusing on cancer research and the optimal use of preventative and integrated treatment interventions to achieve improved outcomes, enhanced survival and quality of life for the cancer patient.
The manuscript management system is completely online and includes a very quick and fair peer-review system, which is all easy to use. Visit http://www.dovepress.com/testimonials.php to read real quotes from published authors. 